\title{
Author Correction: GnRH pulse frequency and irregularity play a role in male aging
}

Zhouguang Wang, Wenhe Wu, Min Soo Kim and Dongsheng Cai (D)

Correction to: Nature Aging https://doi.org/10.1038/s43587-021-00116-5, published online 7 October 2021.

In the version of this article initially published, there was an error in author Wenhe Wu's first name (it was misspelled as Whenhe). This has now been corrected in the online version of the article.

Published online: 20 October 2021

https://doi.org/10.1038/s43587-021-00137-0

๑ The Author(s), under exclusive licence to Springer Nature America, Inc. 2021 